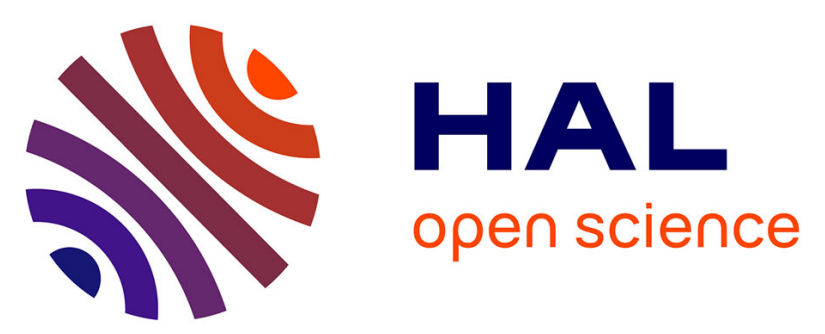

\title{
Electron Transport in Double-Barrier Semiconductor Heterostructures for Thermionic Cooling
}

Xiangyu Zhu, Marc Bescond, Toshiki Onoue, Gerald Bastard, Francesca Carosella, Robson Ferreira, Naomi Nagai, Kazuhiko Hirakawa

\section{- To cite this version:}

Xiangyu Zhu, Marc Bescond, Toshiki Onoue, Gerald Bastard, Francesca Carosella, et al.. Electron Transport in Double-Barrier Semiconductor Heterostructures for Thermionic Cooling. Physical Review Applied, 2021, 16 (6), 10.1103/physrevapplied.16.064017 . hal-03472656

\section{HAL Id: hal-03472656 https://hal.science/hal-03472656}

Submitted on 9 Dec 2021

HAL is a multi-disciplinary open access archive for the deposit and dissemination of scientific research documents, whether they are published or not. The documents may come from teaching and research institutions in France or abroad, or from public or private research centers.
L'archive ouverte pluridisciplinaire HAL, est destinée au dépôt et à la diffusion de documents scientifiques de niveau recherche, publiés ou non, émanant des établissements d'enseignement et de recherche français ou étrangers, des laboratoires publics ou privés. 


\title{
Electron Transport in Double-Barrier Semiconductor Heterostructures for Thermionic Cooling
}

\author{
Xiangyu Zhu, ${ }^{1, *}$ Marc Bescond $\odot,{ }^{2, \dagger}$ Toshiki Onoue $\odot,{ }^{1}$ Gerald Bastard,${ }^{3}$ Francesca Carosella $\odot,{ }^{4}$ \\ Robson Ferreira, ${ }^{3}$ Naomi Nagai, ${ }^{1}$ and Kazuhiko Hirakawa ${ }^{1,2,5, \ddagger}$ \\ ${ }^{1}$ Institute of Industrial Science, University of Tokyo, 4-6-1 Komaba, Meguro-ku, Tokyo 153-8505, Japan \\ ${ }^{2}$ LIMMS/CNRS-IIS, 4-6-1 Komaba, Meguro-ku, Tokyo 153-8505, Japan \\ ${ }^{3}$ Physics Department ENS PSL, Paris Diderot University, 24 Rue Lhomond, 75005 Paris, France \\ ${ }^{4}$ Physics Department ENS PSL, Paris Diderot University, 24 Rue Lhomond, 75005 Paris, France \\ ${ }^{5}$ Institute for Nano Quantum Information Electronics, University of Tokyo, 4-6-1 Komaba, Meguro-ku, \\ Tokyo 153-8505, Japan
}

(Received 12 July 2021; revised 19 October 2021; accepted 12 November 2021; published 8 December 2021)

\begin{abstract}
We investigate electron transport in asymmetric double-barrier $(\mathrm{Al}, \mathrm{Ga}) \mathrm{As} / \mathrm{GaAs}$ thermionic cooling heterostructures. Measurements of temperature-dependent current-voltage characteristics confirm that the dominant electron transport is a sequential process of resonant tunneling injection into and thermionic emission from the quantum-well (QW) cooling layer. The thermal activation energy of the current is found to be strongly dependent on the bias voltage. Furthermore, instead of showing a simple thermal activation behavior, the current exhibits rather complicated temperature and voltage dependence, particularly when the thermionic emission barrier is low. To establish a quantitative understanding, we develop an intuitive analytical model for sequential electron transport that explicitly takes into account scattering effects in the thermionic emission process from the two-dimensional QW states to the three-dimensional above-barrier states. The observed temperature-dependent sequential current is well explained by the present theory.
\end{abstract}

DOI: 10.1103/PhysRevApplied.16.064017

\section{INTRODUCTION}

Progress in ultrahigh-density ultrahigh-speed electronicphotonic devices has come along with the tremendous generation of heat, resulting from thermalization of hot carriers generated by high electric fields. Cooling technologies are, therefore, indispensable for the continuous development of high-performance devices. Solid-state physics may provide relevant alternative approaches to go beyond the standard cooling techniques based on liquid or air (fans) [1]. So far, most of the solid-state solutions rely on the thermoelectric Peltier effect [2-4]. However, thermoelectric devices operate in the near-equilibrium regime and, therefore, the cooling power is limited $[5,6]$. Furthermore, the cooling efficiency deteriorates due to scatteringinduced internal Joule heating. Recently, thermionic cooling, which is based on the thermionic emission process, is attracting considerable attention [7]. The thermionic cooling devices operate far from thermal equilibrium and electron transport is predominantly ballistic [8,9]. A thermionic device structure was proposed by

\footnotetext{
*zxy@iis.u-tokyo.ac.jp

†bescond@iis.u-tokyo.ac.jp

†hirakawa@iis.u-tokyo.ac.jp
}

Mahan [10]. It had two metal parallel plates separated by a short distance (vacuum), which created a potential profile of a single barrier sandwiched by two electrodes [11]. Later, structures in which the vacuum spacer between the two metal plates was replaced with a heterostructured potential barrier were studied [12-17].

More recently, Chao et al. proposed a thermionic cooling structure with asymmetric double barriers [18]. In this structure, a quantum well (QW) is sandwiched between two potential barriers. The first barrier (hereafter, we call it the "emitter" barrier) is designed to be thin and tall. Since the emitter barrier is thin, electrons can tunnel from the emitter electrode into the QW by resonant tunneling, which allows only cold electrons to enter the QW. This selective electron injection is the key for enhancing the cooling efficiency. The second barrier (hereafter, we call it the "collector" barrier) is thick and low. Since the collector barrier is thick, electrons cannot escape the QW by tunneling; instead, they are forced to surpass the collector barrier thermionically (thermionic emission). Indeed, we recently demonstrated that $(\mathrm{Al}, \mathrm{Ga}) \mathrm{As} / \mathrm{GaAs}$ asymmetric double-barrier thermionic cooling structures could reduce the electron temperature, $T_{e}$, from $300 \mathrm{~K}$ down to $250 \mathrm{~K}$ by applying a bias voltage to the structure $[19,20]$. In the asymmetric double-barrier thermionic cooling structures, 
the two-step sequential current that is carried by resonant injection into the QW and subsequent thermionic emission plays an essential role [21]. To improve the cooling performance, an understanding of electron transport and optimization of structural parameters are needed.

Here, we investigate electron transport in asymmetric double-barrier $(\mathrm{Al}, \mathrm{Ga}) \mathrm{As} / \mathrm{GaAs}$ thermionic cooling heterostructures. Measurements of temperature-dependent current-voltage characteristics confirm that the dominant electron-transport process is a sequential process of resonant tunneling injection into and thermionic emission from the QW cooling layer. The thermal activation energy of the current is found to be strongly dependent on the bias voltage. Furthermore, instead of showing a simple thermal activation behavior, the current exhibits rather complicated temperature and voltage dependence, particularly when the thermionic emission barrier is low. To establish a quantitative understanding, we develop an intuitive analytical model for sequential electron transport that explicitly takes into account scattering effects in the thermionic emission process from the two-dimensional QW states to the three-dimensional above-barrier states. The observed temperature-dependent sequential current is well explained by the present theory.

\section{TEMPERATURE-DEPENDENT CURRENT-VOLTAGE CHARACTERISTICS}

The wafers are grown on $n$-type GaAs substrates by using molecular beam epitaxy. We measure several different asymmetric double-barrier thermionic cooling structures and, here, we discuss mainly the following two structures. For sample $A$, we successively grow a 300-nm-thick $n^{ \pm} \mathrm{GaAs}$ emitter layer (Si doping density $=1 \times 10^{18} \mathrm{~cm}^{-3}$ ), a 5 -nm-thick undoped GaAs spacer layer, an undoped 6-nm-thick $\mathrm{Al}_{0.5} \mathrm{Ga}_{0.5} \mathrm{As}$ emitter barrier, an undoped 6-nm-thick GaAs QW, an undoped 100-nm-thick $\mathrm{Al}_{0.25} \mathrm{GaAs}_{0.75} \mathrm{As}$ collector barrier, and a 200-nm-thick $n^{+}$-GaAs collector layer (Si doping density $=1 \times 10^{18} \mathrm{~cm}^{-3}$ ). Sample $B$ has almost the same structure. The only difference is that we replace the collector barrier with an undoped 100 -nm-thick $\mathrm{Al}_{0.2} \mathrm{GaAs}_{0.8} \mathrm{As}$ layer. The band diagram of the sample is schematically illustrated in Fig. 1. The wafer is then photolithographically patterned into mesa structures with various areas, ranging from $80 \times 80$ to $800 \times 800 \mu \mathrm{m}^{2}$. (Au, Ge)Ni/Au contacts are deposited on the front and back sides of the mesas. The samples are finally annealed at $450{ }^{\circ} \mathrm{C}$ under and ambient Ar atmosphere for $5 \mathrm{~s}$. Current densityvoltage $(J-V)$ measurements are performed in a flow-type cryostat.

Figures 2(a) and 2(b), respectively, show the $J-V$ characteristics measured on a mesa of $80 \times 80 \mu \mathrm{m}^{2}$ of sample $A$ at various temperatures, $T$, ranging from 6 to $300 \mathrm{~K}$ (the measurements are performed every $10 \mathrm{~K}$ above $10 \mathrm{~K}$ ), on a linear and a logarithmic scale. In Fig. 2(a), we see clear

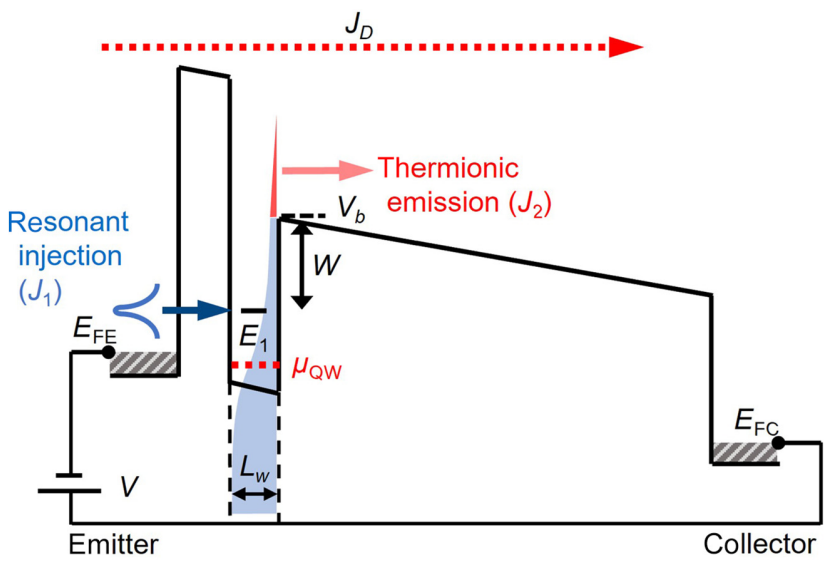

FIG. 1. Band diagram of the asymmetric double-barrier thermionic cooling heterostructure. Electrons are injected from the emitter electrode into the quantum well through the thin and tall emitter barrier (blue arrow) by resonant tunneling $\left(J_{1}\right)$ and are extracted to the collector by the thermionic emission above the thicker barrier (red arrow) $\left(J_{2}\right) . E_{\mathrm{FE}}$ and $E_{\mathrm{FC}}$ are the Fermi levels in the emitter and collector electrodes, respectively. $W$ is the energy difference between the quantized energy state, $E_{1}$, and the collector barrier height, $V_{b} . L_{w}$ is the thickness of the QW. $\mu_{\mathrm{QW}}$ is the quasi-Fermi level in the QW. Current that directly goes from the emitter to the collector ( $J_{D}$; red dotted arrow) does not contribute to cooling, and we do not discuss it in this work.

negative differential $J-V$ curves at low temperatures, where thermal excitation is suppressed, and transport is predominantly due to tunneling. $J$ starts increasing at $V=0.8 \mathrm{~V}$ and shows a sudden decrease at $V=1.3 \mathrm{~V}$. The observed $J-V$ curves are very similar to those of the double-barrier resonant tunneling diodes [22,23]. However, the difference is that, since the collector barrier is thick, only the tunnel leakage current through the collector barrier at high bias voltages is visible, and $J$ does not correctly reflect how many electrons are injected into the QW. Indeed, we see in Fig. 2(b) that electron injection starts at $V$ as low as $0.45 \mathrm{~V}$ and continues up to $1.2 \mathrm{~V}$. The quantized energy state in the QW, $E_{1}$, aligns with the Fermi level in the emitter electrode at $V=0.45 \mathrm{~V}$ and it aligns with the bottom of the conduction band in the emitter at $V=1.2 \mathrm{~V}$. As $T$ is increased, the thermally activated current starts to increase. For $V<0.45 \mathrm{~V}, J$ increases by a factor as much as $10^{7}-10^{8}$, when $T$ is increased from 6 to $300 \mathrm{~K}$. This is in strong contrast with $J$ in the tunnel-leakage region $(V>1.0 \mathrm{~V})$, where the increase in $J$ with respect to $T$ is less than 1 order of magnitude. From the observed $J-V$ curves, we can tell that the maximum resonant tunneling injection from the emitter into the QW occurs at $V=1.0-1.2 \mathrm{~V}$. For larger $V$, the quantized energy state in the QW goes below the emitter conduction band, leading to a sudden decrease in $J$.

Similarly, Figs. 2(c) and 2(d) show the $J-V$ characteristics of sample $B$ on a linear and a logarithmic scale, respectively. The $J-V$ curves for sample $B$ are similar to 

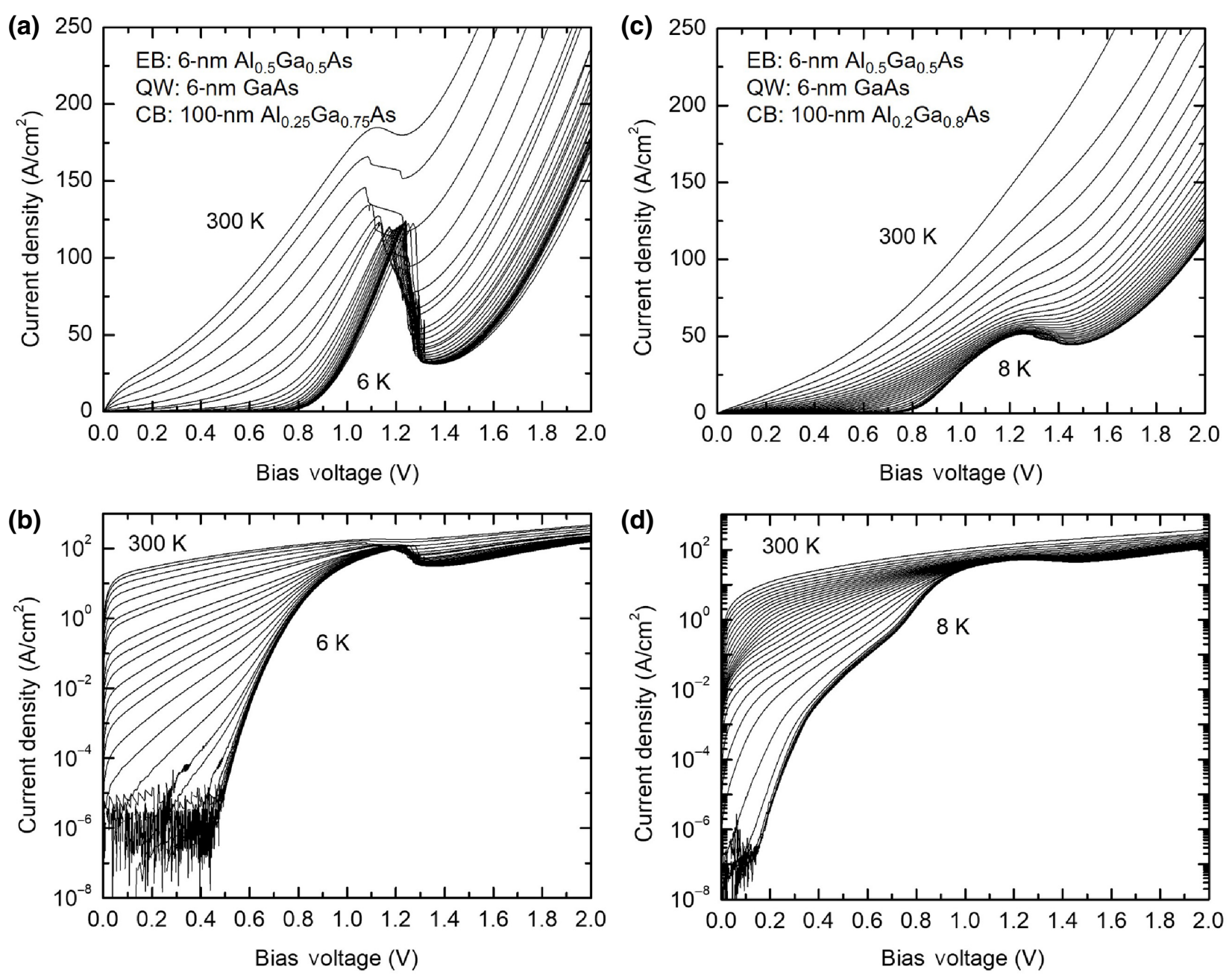

FIG. 2. Current density, $J$, measured as a function of bias voltage, $V$, at various temperatures from less than $10 \mathrm{~K}$ to $300 \mathrm{~K}$. Above $10 \mathrm{~K}, J-V$ curves are measured every $10 \mathrm{~K}$. Sample $A$, (a) linear scale; (b) logarithmic scale. Sample $B$, (c) linear scale; (d) logarithmic scale.

those of sample $A$, although the negative differential conductance feature is less sharp. This may originate from the fact that the collector barrier of sample $B$ under bias voltage is rather leaky. On a linear scale, $J$ starts increasing at $V \sim 0.8 \mathrm{~V}$ and shows a small decrease at $V \sim 1.3 \mathrm{~V}$. On a logarithmic scale [Fig. 2(d)], we can see a finite current, even at a voltage as low as about $0.2 \mathrm{~V}$, reflecting the lower collector barrier height.

Figure 3(a) plots $J$ measured on sample $A$ as a function of $1 / T$ at various $V$. The observed exponential increase in $J$ for $T>60 \mathrm{~K}$ indicates that the current is thermally activated, while $J$ becomes almost temperature independent for $T<60 \mathrm{~K}$, showing that the current is carried predominantly by tunneling. From the slope of the $J$ versus $1 / T$ plot, the thermal activation energy, $E_{a}$, can be determined. $E_{a}$ determined at $V=0.1 \mathrm{~V}$ is $180 \mathrm{meV}$ and gradually decreases to $65 \mathrm{meV}$ at $V=0.8 \mathrm{~V}$. From the simple band structure shown in Fig. 1, it is expected that $E_{a}$ is equal to the energy difference, $W$, between the quantized energy state in the QW, $E_{1}$, and the top of the collector barrier, $V_{b}$. In the present sample, $W$ is $135 \mathrm{meV}$. Surprisingly, the experimentally determined $E_{a}$ is strongly bias dependent and very different from $W$.

Figure 3(b) shows $J$ measured on sample $B$ as a function of $1 / T$ at various $V$. As seen in the figure, the $J-1 / T$ curve shows rather complicated bias and $T$ dependence; at $V=0.05 \mathrm{~V}$, the $J-1 / T$ curve exhibits two slopes (a steeper slope at higher $T$ and a smaller negative slope at lower $T$ ), with an intermediate temperature region from 250 to $120 \mathrm{~K}$. The observed complicated behavior gradually disappears at higher bias voltages. We discuss the $T$ dependence of $J$ in Sec. IV.

\section{THEORETICAL FORMULATION FOR TWO-STEP SEQUENTIAL CURRENT}

To understand the observed temperature dependence of $J$, we develop an intuitive analytical theory describing 


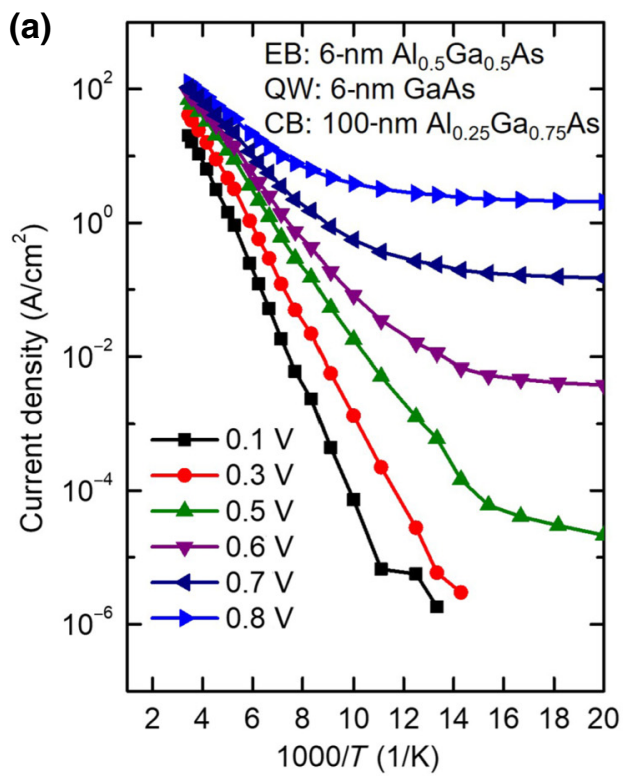

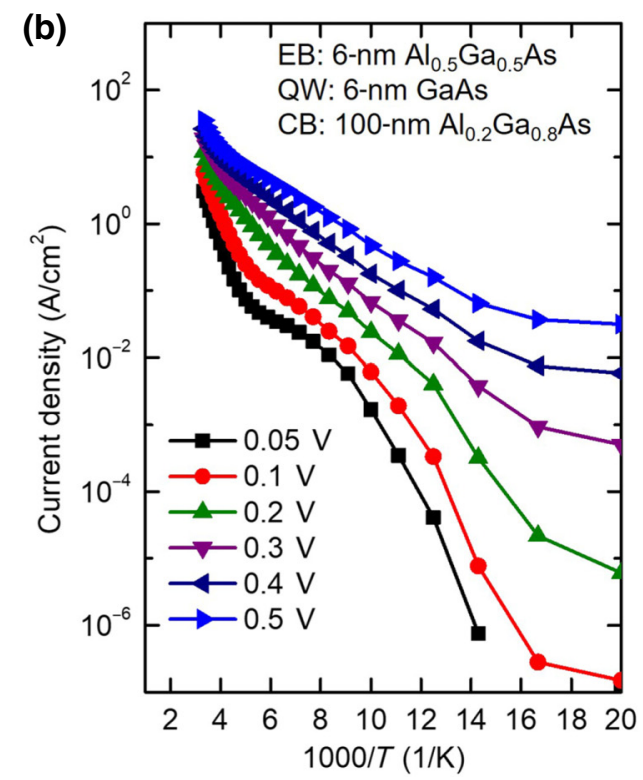

FIG. 3. Current densities measured at various $V$ plotted as a function of $1 / T$. (a) Sample $A$, (b) sample $B$. electron transport in the asymmetric double-barrier thermionic cooling structure (shown in Fig. 1) [24,25]. Electron transport from the emitter to the collector can be classified into two current components: one is a current directly carried from the emitter to the collector (we call this component $J_{D}$ ) and the other is a current component that is carried by resonant electron injection into the QW and subsequent thermionic emission (we call this component $\left.J_{S}\right)$. $J_{D}$ can easily be calculated by using, for example, the transfer-matrix method [26], and in our asymmetric double-barrier structures $J_{D}$ is usually much smaller than $J_{S}$. Therefore, we do not discuss $J_{D}$ here.

Theoretical treatment of $J_{S}$ is not trivial for two reasons: (1) we need to properly treat resonant tunneling and thermionic emission in such a way that current continuity throughout the structure is fulfilled, and (2) we need to express thermionic emission from the two-dimensional (2D) QW to the three-dimensional (3D) above-barrier states. Let us express $J_{S}$ in terms of the resonant injection current, $J_{1}$, and thermionic emission current, $J_{2}$. We use the quasi-Fermi level in the $\mathrm{QW}, \mu_{\mathrm{QW}}$, as a parameter to describe the number of electrons in the quantized energy state, $E_{1}$. Since $\mu_{\mathrm{QW}}$ is the common parameter for determining $J_{1}$ and $J_{2}$, we calculate $\mu_{\mathrm{QW}}$ self-consistently in such a way that the current conservation across the entire structure is fulfilled, i.e., $J_{1}=J_{2}$. In the following, we describe our theory in more detail.

\section{A. Electron injection by resonant tunneling}

Considering the sequential description of the current shown in Fig. 1, we calculate in this subsection the resonant injection component, $J_{1}$, using the formulation developed for sequential resonant tunneling [27]. $J_{1}$ is expressed as

$$
\begin{aligned}
J_{1} & =-e \sum_{k} v_{z}\left(f_{E}-f_{\mathrm{QW}}\right) A\left(E_{z}\right) \Gamma\left(E_{z}\right), \\
\frac{\Gamma\left(E_{z}\right)}{\hbar} & =\frac{v_{z}}{2 L_{w}} T\left(E_{z}\right), A\left(E_{z}\right) \\
& =\frac{\Gamma\left(E_{z}\right)}{\left(E_{z}-E_{1}\right)^{2}+\left[\Gamma\left(E_{z}\right) / 2\right]^{2}},
\end{aligned}
$$

where $e$ is the elementary charge; $\hbar$ is the reduced Planck constant; $E_{1}$ is the quantization energy in the QW; and $f_{E}$ and $f_{\mathrm{QW}}$ are the electron-distribution functions in the emitter electrode and QW, respectively. $\Gamma / \hbar$ defines the tunneling rate at which an electron in the QW tunnels through the emitter barrier and is determined by the electron velocity, $v_{z}$, in the $\mathrm{z}$ direction (perpendicular to the heterointerfaces) at $E_{1}$ and the transmission probability, $T\left(E_{z}\right)$, of the emitter barrier. $L_{w}$ is the thickness of the QW. $A\left(E_{z}\right)$ expresses the homogeneous broadening of the quantized energy level in the QW due to tunnel leakage.

We consider the emitter electrode to have 3D physical properties and perform integration in the in-plane direction and the $z$ direction:

$$
\begin{aligned}
J_{1} & =-\frac{2 e}{8 \pi^{3}} \iint d k_{\|}^{2} d k_{z} v_{z}\left(f_{E}-f_{\mathrm{QW}}\right) A\left(E_{z}\right) \Gamma\left(E_{z}\right), \\
& =-\frac{e m^{*}}{2 \pi^{2} \hbar^{3}} \iint d E_{\|} d E_{z}\left(f_{E}-f_{\mathrm{QW}}\right) A\left(E_{z}\right) \Gamma\left(E_{z}\right) .
\end{aligned}
$$

Here, $m^{*}$ is the electron's effective mass. Assuming the Boltzmann-distribution function and that the Lorentzian function, $A\left(E_{z}\right)$, is sharp enough to be described by a delta function, we can directly integrate Eq. (3) in the $z$ direction 
and obtain

$$
J_{1}=-\frac{e m^{*} k_{B} T}{\pi \hbar^{3}} \Gamma\left(E_{1}\right)\left[e^{-\beta\left(E_{1}-E_{\mathrm{FE}}\right)}-e^{-\beta\left(E_{1}-\mu_{\mathrm{QW}}\right)}\right] .
$$

Here, $k_{B}$ is the Boltzmann constant, $T$ is the temperature, and $\beta=\frac{1}{k_{B} T}$.

\section{B. Scattering-Assisted thermionic emission from quantum wells}

Next, we calculate the thermionic emission current, $J_{2}$, extracted from the 2D QW towards the 3D above-barrier states. The transition of electrons from a $2 \mathrm{D}$ system to a $3 \mathrm{D}$ system is not trivial. Here, we present our scatteringassisted thermionic emission theory.

To describe how the 2D-confined electrons in the QW acquire a velocity component in the $z$ direction, we develop a formulation that takes into account scattering effects in the thermionic emission process from the QW. The details of the derivation can be found in the Appendix A and B. We considered three scattering mechanisms, namely, longitudinal-optical (LO) phonon scattering (Fröhlich coupling), longitudinal-acoustic (LA) phonon scattering (deformation potential coupling), and Coulombic impurity scattering. We find that the thermionic emission current assisted by LO phonon scattering is dominant; the LO phonon-assisted current is 1 order of magnitude larger than the LA phonon-assisted current and the Coulombic impurity scattering is almost negligible, unless the QW is heavily doped. The dominant LO phononassisted thermionic emission current from the 2D QW to the $3 \mathrm{D}$ above-barrier states is expressed as follows:

$$
\begin{aligned}
J_{2}= & \frac{-e k_{B} T}{S L_{w} 16 \pi \hbar \delta} n_{e} \frac{e^{2}}{\varepsilon_{0}} \hbar \omega_{\mathrm{LO}}\left[\frac{1}{\varepsilon_{r}(\infty)}-\frac{1}{\varepsilon_{r}(0)}\right] \\
& \times e^{-\beta\left(V_{b}-E_{1}-\hbar \omega_{\mathrm{LO})}\right.} n_{\mathrm{LO}} \operatorname{Int} \\
\text { Int } \approx & \frac{1}{V_{b}-E_{1}-\hbar \omega_{\mathrm{LO}}}+\frac{e^{-\beta \hbar \omega_{\mathrm{LO}}}}{V_{b}-E_{1}+\hbar \omega_{\mathrm{LO}}} .
\end{aligned}
$$

Here, $\hbar \omega_{\text {LO }}$ is the LO phonon energy; $\delta$ is the broadening parameter (in this work, we set $\delta=5 \mathrm{meV}$ ); $S L_{w}$ is the volume of the active QW region; $\varepsilon_{r}(0)$ and $\varepsilon_{r}(\infty)$ are the permittivities at low and high frequencies, respectively. $n_{\mathrm{LO}}$ is the Bose occupation number for LO phonons. $V_{b}$ is the collector barrier height. Notably, $J_{2} \propto n_{\mathrm{LO}} e^{-\beta\left(V_{b}-\mu_{\mathrm{QW}}-\hbar \omega_{\mathrm{LO}}\right)}$, where $n_{\mathrm{LO}}=1 /\left(e^{\beta \hbar \omega_{\mathrm{LO}}}-1\right)$. Therefore, the LO phonon-assisted thermionic current has a smaller activation energy by $\hbar \omega_{\mathrm{LO}}$ at high temperatures, when compared with the conventional Richardson equation [28]. As the temperature becomes lower and $e^{\beta \hbar \omega_{\mathrm{LO}}}$ becomes much larger than unity, the activation energy for $J_{2}$ is reduced to the one for Richardson theory, i.e., $V_{b}-\mu_{\mathrm{QW}}$. Derivations and expressions for thermionic emission from the 2D QW to the 3D above-barrier states assisted by other scattering mechanisms are also found in the Appendix C and D.

\section{THERMAL ACTIVATION BEHAVIOR OF CURRENT}

Using the analytical theory described in Sec. III, we calculate $J$ for the fabricated thermionic cooling structure described in Sec. II. Figure 4(a) shows the temperature dependence of $J$ calculated for sample $A$ at $V=0.1 \mathrm{~V}$ by using the theory for LO phonon-assisted thermionic emission (red). In the same figure, $J$ measured at $V=0.1 \mathrm{~V}$ is replotted from Fig. 3(a) as black dots. Notably, overall agreement between theory and experiment is reasonable, even though no fitting parameters, except for the broadening parameter, $\delta(=5 \mathrm{meV})$, are used in the calculation.

Figure 5(a) plots the thermal activation energy of the current determined for sample $A$ at $T \sim 220 \mathrm{~K}$ from experiment (blue dots) as a function of $V$. The energy difference, $W=V_{b}-E_{1}$, expected from the sample design (green) is also plotted. $W$ is almost independent of $V$, while the observed activation energy decreases as $V$ is increased. The small variation in $W$ as a function of $V$ is due to the Stark shift in the thin QW. In the same figure, we plot the activation energy calculated by using the LO phonon-assisted thermionic emission theory (blue). The activation energy predicted by theory is in reasonable agreement with experiment, indicating that the present analytical theory involves the correct physics and provides a quantitative account of sequential electron transport of resonant injection into and thermionic emission from the 2D QW.

Another intriguing feature in Fig. 4(a) is the deviation of $J$ from simple thermally activated behavior when $T>200 \mathrm{~K} . J$ indeed shows a complicated temperature dependence; i.e., a bending and a sharper increase as $T$ is increased. This behavior arises from a strong temperature dependence of $\mu_{\mathrm{QW}}$. In Fig. 4(b), $\mu_{\mathrm{QW}}$ calculated by using phonon-assisted thermionic emission theory is plotted as a function of $1 / T$ (green). The origin of the vertical axis is taken to be the Fermi level in the emitter electrode at $T=0 \mathrm{~K}$. The black and orange curves show the Fermi levels in the emitter and the collector, respectively. As seen in the figure, calculated $\mu_{\mathrm{QW}}$ moves from the Fermi level in the collector to the Fermi level in the emitter as $T$ is decreased. This evolution can be understood by considering the temperature dependence of the resistances of the emitter and collector barriers. Electron transport across the emitter barrier is predominantly tunneling, which is basically temperature independent. On the other hand, electron transport across the collector barrier is mainly due to thermionic emission at low voltages and 

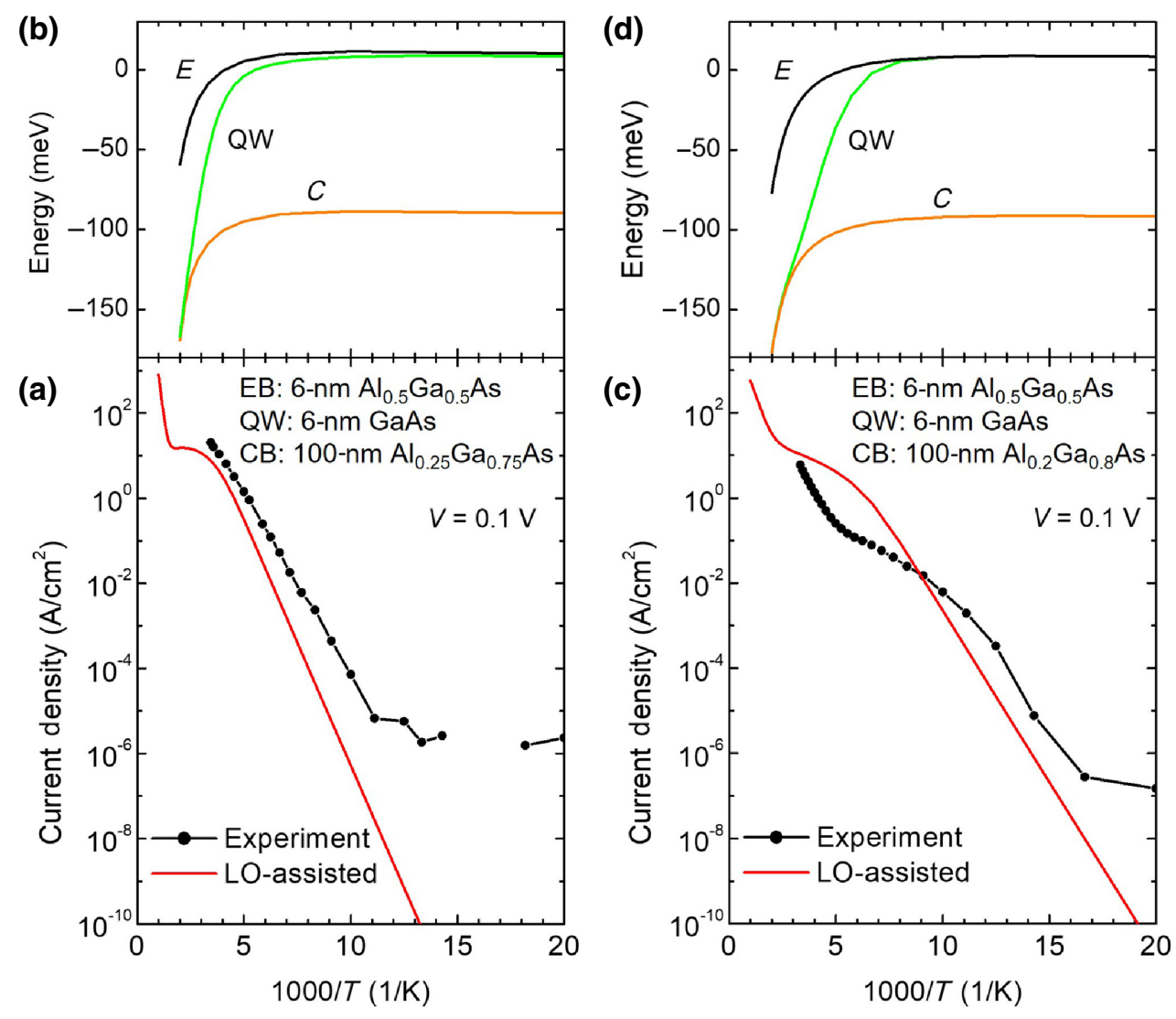

FIG. 4. (a) Current density, $J$, measured at $V=0.1 \mathrm{~V}$ is plotted as a function of $1 / T$ (black dots) replotted from Fig. 3(a). Measured $J$ is compared with LO phonon-assisted thermionic emission theory (red). (b) Quasi-Fermi level in the quantum well, $\mu_{\mathrm{QW}}$, under bias voltage $V=0.1 \mathrm{~V}$ calculated by using LO phonon-assisted thermionic emission theory as a function of $1 / T$ (green). $T$ dependence of Fermi levels in the emitter (black) and the collector (orange) are also plotted. (c) $J$ measured at $V=0.1 \mathrm{~V}$ is plotted as a function of $1 / T$ (black dots) replotted from Fig. 3(b). Measured $J$ is compared with the LO phonon-assisted thermionic emission theory (red). (d) $\mu_{\mathrm{QW}}$ under bias voltage $V=0.1 \mathrm{~V}$ calculated by using LO phonon-assisted thermionic emission theory is plotted as a function of $1 / T$ (green). $T$ dependence of Fermi levels in the emitter (black) and the collector (orange) are also plotted.

has a very strong temperature dependence; the resistance of the collector barrier rapidly increases with decreasing $T$. Since $\mu_{\mathrm{QW}}$ is determined in such a way that current continuity is fulfilled throughout the entire structure, $\mu_{\mathrm{QW}}$ is close to the Fermi level of the low-resistance collector side at high temperatures and gradually moves to the emitter Fermi level side as $T$ is reduced. The complicated behavior in the $T$-dependent current appears in such a transition region.

As seen in Fig. 4(c), such behavior is more clearly observed in sample $B$. Sample $B$ has a lower collector barrier than that of sample $A$ and the transition in $\mu_{\mathrm{QW}}$ is expected to occur at lower temperatures. The black dots in Fig. 4(c) represent $J$ measured at $V=0.1 \mathrm{~V}$ on sample $B$, which are replotted from Fig. 3(b). Calculated $T$-dependent current density is plotted in red. As shown in Fig. 4(d), when $T<150 \mathrm{~K}, \mu_{\mathrm{QW}}$ is attached to the Fermi level in the emitter and the predicted activation energy has a constant value, i.e., $E_{a}=V_{b}-\mu_{\mathrm{QW}}$. As a result, we see straight curves of calculated $J$ for $T<150 \mathrm{~K}$. When $T>300 \mathrm{~K}$, calculated $\mu_{\mathrm{QW}}$ is related to the Fermi level in the collector and $J$ exhibits a steeper slope. In Fig. 5(b), we compare the activation energies determined for sample $B$ for low- $(T<100 \mathrm{~K})$ and high-temperature regions $(T>200 \mathrm{~K})$ with those predicted by the present theory at various bias voltages. The activation energies at $T \sim 70 \mathrm{~K}$ show similar behavior to those of sample $A$, but are slightly smaller, due to the lower collector barrier height. However, at $T \sim 220 \mathrm{~K}$ (red dots), the activation energy rapidly increases with decreasing $V$. This behavior is also reproduced by the present theory (red curve). This is because $\mu_{\mathrm{QW}}$ is attached to the collector Fermi level and is much lower than $E_{1}$. As $V$ is increased, tunnel leakage through the collector barrier sets in and the collector barrier becomes less resistive, which leads to a reduction in $E_{a}$. Although the agreement between theory and experiment is not perfect, the present theory reasonably reproduces such a phenomenon. 


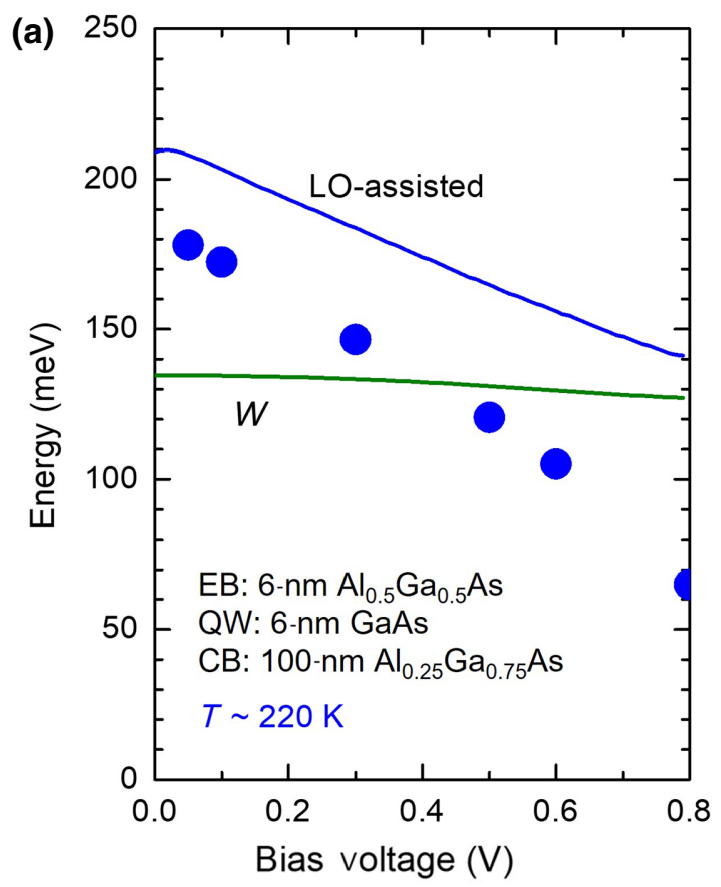

\section{SUMMARY AND OUTLOOK}

We investigate electron transport in asymmetric double-barrier (Al, Ga)As/GaAs thermionic cooling heterostructures. Measurements of temperature-dependent current-voltage characteristics confirm that the dominant electron-transport process is a sequential process of resonant tunneling injection into and thermionic emission from the QW cooling layer. The thermal activation energy of the current is found to be strongly dependent on the bias voltage. Furthermore, instead of showing simple thermal activation behavior, the current exhibits rather complicated temperature and voltage dependence, particularly when the thermionic emission barrier is low. To establish a quantitative understanding, we develop an intuitive analytical model for sequential electron transport that explicitly takes into account scattering effects in the thermionic emission process from the two-dimensional QW states to the three-dimensional above-barrier states. The observed temperature-dependent sequential current is well explained by the present theory.

The present asymmetric double-barrier heterostructures show excellent cooling capabilities for electrons [19] but a rather weak reduction of lattice temperature [20]. The physical origin of such different cooling behavior lies in the huge difference in heat capacitances for electrons and phonons. It is clear from the theory that we have developed that a straightforward way to cool the phonon system more efficiently would be to have more electrons at our disposal. In our samples fabricated by using GaAs-based materials, there are typically multiples of $10^{10} \mathrm{~cm}^{-2}$ electrons in the
FIG. 5. Thermal activation energy determined from the $J-1 / T$ curves as a function of bias voltage. Sample $A$, (a) calculated around $220 \mathrm{~K}$ (blue dots). Sample $B$, (b) calculated around $70 \mathrm{~K}$ (blue dots) and $250 \mathrm{~K}$ (red dots). $W$ is the energy difference between the quantized energy level in the quantum well and the top of the collector barrier (green). Thermal activation energy calculated by using the LO-phonon-assisted thermionic emission is also plotted. Sample $A$, (a) calculated around $220 \mathrm{~K}$ (blue line). Sample $B$, (b) calculated around $70 \mathrm{~K}$ (blue line) and $250 \mathrm{~K}$ (red line).
QW. An enhancement in the QW population by factors of 10 can be envisioned by doping.

Since cooling the lattice system still appears to be elusive in semiconductor-based structures, the present asymmetric double-barrier cooling structures may be more useful in devices in which electronic cooling plays an important role. Light-emitting devices will gain better efficiency if the carrier temperature can be decreased via our cooling device by $30-50 \mathrm{~K}$. Nonradiative losses due to thermal escape of carriers outside the confining potential wells would be decreased. Similarly, in QW infrared photodetectors, the dark current will be reduced by cooling electrons in the QW. These are only a few examples. We believe there will be many more useful applications of electron cooling.

\section{ACKNOWLEDGMENTS}

We thank Koung-An Chao, Chloe Salhani, and Guillaume Dangoisse for fruitful discussions. This work is supported by KAKENHI from the JSPS (Grants No. JP19K21957 and No. JPJSCCA20190006) and by the GELATO ANR project (Grant No. ANR-21-CE50-0017).

\section{APPENDIX A: CALCULATING THERMIONIC EMISSION FROM THE TWO-DIMENSIONAL QUANTUM WELL TO THREE-DIMENSIONAL STATES}

The Richardson approach to the thermionic current applied to a QW assumes that all electrons are thermalized among themselves, including extended states along $z$. 
It leads, after thermal averaging, to a current of

$$
\begin{aligned}
J_{2} & =\left\langle J_{z}\right\rangle=-\frac{e m^{*}}{2 \pi^{2} \hbar^{3}}(k T)^{2} e^{\frac{-\left(V_{b}-\mu\right)}{k T}} \\
& =-e n_{3 d} \sqrt{\frac{\pi k T}{2 m *}} \exp \left(-\frac{V_{b}}{k T}\right),
\end{aligned}
$$

where $-e$ is the electron charge, $V_{b}$ is the barrier height, and $n_{3 \mathrm{D}}=n_{2 \mathrm{D}} / L_{w}$ is the $3 \mathrm{D}$ volume density of electrons in the QW of thickness $L_{w}$. An important drawback of this $3 \mathrm{D}$ model is that it neglects the size quantization along the $z$ direction and its allowance of any kinetic energy $\left(<\right.$ or $\left.\geq V_{b}\right)$ for the electron's $z$ motion while the QW thickness is only $6 \mathrm{~nm}$. Deeply rooted in a bulk description, we note that Eq. (A1) lacks any length parameter (in particular, the QW thickness) and any characteristic energy, $E_{1}$.

A remarkable feature of the Richardson formula is that, once an electron has enough kinetic energy along $z$, it will contribute to the current and, on the contrary, if an electron has a total kinetic energy larger than $V_{b}$, but a longitudinal kinetic energy smaller than $V_{b}$, it gives a zero contribution to $\left\langle J_{z}\right\rangle$. Finally, the assumption of thermalization between the electrons bound to the QW and those in the extended states is unrealistic for such a narrow slab of material $(6 \mathrm{~nm})$, since the time spent in the well for a delocalized electron is much smaller than any reasonable thermalization time.

Our idea for the thermionic current in semiconductor heterostructures consists of remarking that, in contrast to the Richardson model, these are the defects, impurities, and phonons that are at the origin of the current. There are thermalized electrons bound to the QW (in the $E_{1}$ bound state for their $z$ motion) that cannot alone contribute to the current (because there is no current for a bound state). Some of these electrons have a total energy larger than $V_{b}$. Then, scattering with phonons, impurities, etc. will convert part of their (large) in-plane kinetic energy into longitudinal kinetic energy and lead to a nonzero $J_{2}=\left\langle J_{z}\right\rangle$.

\section{APPENDIX B: THERMIONIC CURRENT ASSOCIATED WITH LO PHONON EMISSION OR ABSORPTION}

We use a perturbative approach to compute the scattering-induced thermionic current. For the phonons, we take a bulklike approach for simplicity. The LO branch is assumed to be dispersionless and the electron-phonon interaction is the Fröhlich coupling:

$$
H_{e-\mathrm{ph}}=\sum_{\vec{Q}_{l}} \frac{-i C_{F}}{Q_{l}}\left[a_{\vec{Q}_{l}}^{\dagger} \exp \left(-i \overrightarrow{Q_{l}} \cdot \vec{r}\right)+a_{\vec{Q}_{l}} \exp \left(i \overrightarrow{Q_{l}} \cdot \vec{r}\right)\right]
$$

where $C_{F}$ is the Fröhlich constant:

$$
\Omega C_{F}^{2}=\frac{e^{2} \hbar \omega_{\mathrm{LO}}}{2 \varepsilon_{0}}\left[\frac{1}{\varepsilon_{r}(\infty)}-\frac{1}{\varepsilon_{r}(0)}\right],
$$

and $\Omega=S L_{w}$ is the volume of the crystal. $a_{\vec{Q}_{l}}^{\dagger}$ and $a_{\vec{Q}_{l}}$ are the creation and annihilation operators, respectively, of a LO phonon with wavevector $\vec{Q}_{l}$. The complete Hamiltonian is

$$
\begin{aligned}
H= & H_{\mathrm{el}}+\sum_{\overrightarrow{Q_{l}}}\left(n_{\vec{Q}_{l}}+\frac{1}{2}\right) \hbar \omega_{\mathrm{LO}} \\
& +H_{e-\mathrm{ph}} ; \quad n_{\vec{Q}_{l}}=0,1,2, \ldots
\end{aligned}
$$

The phonon states are labeled by $\left|n_{\vec{Q}_{l}}\right\rangle$. The eigenstates of the decoupled electrons and phonons are written as

$$
\begin{aligned}
|\psi\rangle & =\left|\varphi_{\mathrm{el}}\right\rangle \otimes \prod_{\vec{Q}_{l}}\left|n_{\vec{Q}_{l}}\right\rangle, \text { where }\left|\varphi_{\mathrm{el}}\right\rangle \\
& =\left|\chi_{1}, k_{\mathrm{par}}\right\rangle \text { or }\left|q_{z}, k_{\mathrm{par}}\right\rangle, \quad q_{z}>0
\end{aligned}
$$

For simplicity, we consider a single QW structure of total thickness $L_{w}$ and assume a structureless continuum, i.e., $\langle z| q_{z}>=\left(1 / \sqrt{L_{w}}\right) \exp \left(i q_{z} z\right), q_{z}>0$. Then, to first order in $H_{e-\mathrm{ph}}$, there is

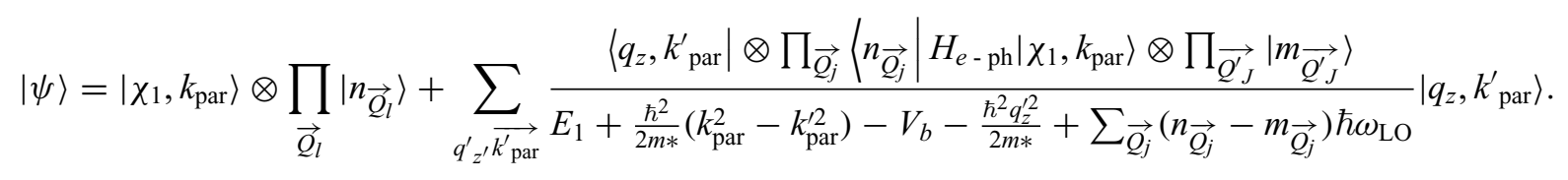

Now we can compute the current. There will be

(1) a zero in the $H_{e-\mathrm{ph}}$ term that vanishes identically because there is no current for a bound state;
(2) two terms linear in $H_{e-\mathrm{ph}}$ that vanish identically because the phonon numbers in the two terms are different; and

(3) a term that is quadratic in $H_{e-\mathrm{ph}}$; thus, we get, for one spin direction, 


$$
\begin{aligned}
\left\langle J_{z}\right\rangle= & \frac{-e}{S L_{w}} C_{F}^{2} \exp \left[-\beta\left(E_{1}-\mu\right)\right] \sum_{\overrightarrow{\mathrm{par}}, k_{\mathrm{par}}^{\prime}} \sum_{q_{z}^{\prime}, Q_{l z}} \exp \left(-\beta \frac{\hbar^{2}}{2 m *} k_{\mathrm{par}}^{2}\right) \Theta\left(E_{1}+\frac{\hbar^{2}}{2 m *} k_{\mathrm{par}}^{2}-V_{b}\right) \\
& \times \frac{\hbar q_{z}^{\prime}}{m *} \frac{\left|\left\langle q_{z}^{\prime}\left|\exp \left(-i Q_{l z} z\right)\right| \chi_{1}\right\rangle\right|^{2}}{Q_{l z}^{2}+\left(\overrightarrow{k_{\mathrm{par}}^{\prime}}-\overrightarrow{k_{\mathrm{par}}}\right)^{2}}\left\{\frac{n \vec{Q}_{l}}{\left[E_{1}+\hbar \omega_{\mathrm{LO}}+\frac{\hbar^{2}}{2 m *}\left(k_{\mathrm{par}}^{2}-k_{\mathrm{par}}^{\prime 2}\right)-V_{b}-\frac{\hbar^{2} q_{z}^{\prime 2}}{2 m *}\right]^{2}}\right. \\
& \left.+\frac{n \vec{Q}_{l}}{\left[E_{1}-\hbar \omega_{\mathrm{LO}}+\frac{\hbar^{2}}{2 m *}\left(k_{\mathrm{par}}^{2}-k_{\mathrm{par}}^{\prime 2}\right)-V_{b}-\frac{\hbar^{2} q_{z}^{\prime 2}}{2 m *}\right]^{2}}\right\},
\end{aligned}
$$

where $\Theta(x)$ is the Heaviside step function $[\Theta(x<0)=0$, $\Theta(x>0)=1]$.

We have two terms that correspond, respectively, to the absorption of a $\mathrm{LO}$ phonon (proportional to $n_{\mathrm{LO}}$ ) and to the emission of a LO phonon (proportional to $1+n_{\mathrm{LO}}$ ). Absorption is unfavored because one needs to get available phonons, but it is favored by the larger thermal population of electrons. There exists another contribution to $\left\langle J_{z}\right\rangle$ that arises from the second-order correction in $H_{e-\mathrm{ph}}$ to the wave function. It is very cumbersome to handle. Fortunately, numerical calculations show its magnitude is about 1/10 of Eq. (B6).

There exist divergencies at the resonances (vanishing denominators) in Eq. (B6). These divergencies are removed by putting a $\delta^{2}$ where $\delta$ empirically takes care of the broadening of the continuum and QW-bound states. Summarizing the evaluation of Eq. (B6), summing over spin direction, we write the average current as a materialdependent constant multiplied by integrals (of the exponential integral type), as follows:

$$
\begin{aligned}
\left\langle J_{z}\right\rangle= & \frac{-e k_{B} T}{L_{w} 16 \pi \hbar \delta} \frac{n_{e}}{S} \frac{e^{2}}{\varepsilon_{0}} \hbar \omega_{\mathrm{LO}}\left[\frac{1}{\varepsilon_{r}(\infty)}-\frac{1}{\varepsilon_{r}(0)}\right] \\
& \times e^{-\beta\left(V_{b}-E_{1}-\hbar \omega_{\mathrm{LO})}\right.} n_{\mathrm{LO}} \mathrm{Int},
\end{aligned}
$$

where $n_{e} / S$ is the areal concentration of electrons in the QW:

$$
n_{e}=\frac{m * S k_{B} T}{\pi \hbar^{2}} \exp \left[-\beta\left(E_{1}-\mu\right)\right]
$$

and

$$
\begin{aligned}
\text { Int }= & \int_{0}^{\infty} d t e^{-t} \frac{1}{\left|V_{b}-E_{1}-\hbar \omega_{\mathrm{LO}}+t k_{B} T\right|} \\
& +e^{-\beta \hbar \omega_{\mathrm{LO}}} \int_{0}^{\infty} d t e^{-t} \frac{1}{\left|V_{b}-E_{1}+\hbar \omega_{\mathrm{LO}}+t k_{B} T\right|}
\end{aligned}
$$

$$
\operatorname{Int} \approx \frac{1}{V_{b}-E_{1}-\hbar \omega_{\mathrm{LO}}}+\frac{e^{-\beta \hbar \omega_{\mathrm{LO}}}}{V_{b}-E_{1}+\hbar \omega_{\mathrm{LO}}}
$$

We find that the current is proportional to the electron concentration with the right energy (either above the threshold for LO emission or LO absorption) and is therefore thermally activated. In contrast to the Richardson law, we find an expression that is not universal, since $\left\langle J_{z}\right\rangle$ depends on the strength of the Fröhlich coupling and is proportional to the number of available LO phonons. Making numbers appropriate to our sample, we find that the scattering-induced thermionic current is of the same order of magnitude as the one predicted by using the Richardson law.

\section{APPENDIX C: THERMIONIC CURRENT ASSOCIATED WITH ACOUSTIC PHONON EMISSION OR ABSORPTION}

We compute the thermionic current due to the interaction between electrons and LA phonons. For the phonons, we take a bulklike approach for simplicity. The LA branch is assumed to display a linear dispersion and the electronphonon interaction is the deformation potential coupling given by

$H_{e-\mathrm{ph}}=\sum_{\overrightarrow{Q_{l}}} \alpha_{\mathrm{LA}}(Q)\left[a_{\vec{Q}_{l}}^{+} \exp \left(-i \overrightarrow{Q_{l}} \cdot \vec{r}\right)+a_{\vec{Q}_{l}} \exp \left(i \overrightarrow{Q_{l}} \cdot \vec{r}\right)\right]$

where $\alpha_{\mathrm{LA}}$ is the deformation potential constant given by

$$
\alpha_{\mathrm{LA}}^{2}=\frac{D^{2}}{2 \rho c_{s}^{2} \Omega} \hbar c_{s} Q
$$

where $D=7.2 \mathrm{eV} ; c_{s}$ is the sound velocity, which is assumed to be isotropic; and $\rho$ is the density. We proceed in the evaluation of $\left\langle J_{z}\right\rangle$ by neglecting the LA phonon energies, $\hbar c_{s} Q$, compared with electron energies, such as 
$V_{b}-E_{1}$. Moreover, the occupation function, $n_{\mathrm{LA}}$, is approximated by its high-temperature limit $\left(k_{B} T / \hbar c_{s} Q\right)$. Finally, we end up with

$$
\left\langle J_{z}\right\rangle=\frac{-e}{L_{w}} \frac{n_{e}}{S} e^{-\beta\left(-E_{1}+V_{b}\right)} \frac{D^{2}}{4 \rho c_{s}^{2}}\left(k_{B} T\right)^{2} \frac{m^{*}}{\pi \delta \hbar^{3}} .
$$

We find again thermally activated behavior, but with a $T^{2}$ prefactor, instead of $T$ like in Eq. (B7). This is because we use the high-temperature limit of $n_{\mathrm{LA}}$. At room temperature, with parameters adapted to our structure, we find that the acoustic phonon-scattering contribution to $\left\langle J_{z}\right\rangle$ is about $7 \%$ of that of the LO phonon contribution.

\section{APPENDIX D: THERMIONIC CURRENT ASSOCIATED WITH INTERACTIONS WITH IONIZED IMPURITIES}

We consider planar doping at $z=z_{0}$ by $N_{\text {imp }}$ randomly distributed ionized impurities. Proceeding as before, we find

$\left\langle J_{z}\right\rangle=-\frac{e \hbar}{S L_{w}} \frac{n_{e} e^{-\beta\left(V_{b}-E_{1}\right)}}{16 \pi^{2} \delta m^{*}} \frac{\left(k_{B} T\right)}{\left(V_{b}-E_{1}\right)^{2}} \frac{N_{\mathrm{imp}}}{S}\left(\frac{e^{2}}{\varepsilon_{0} \varepsilon_{r}}\right)^{2} \chi_{1}^{2}\left(z_{0}\right)$.

For $N_{\mathrm{imp}} / S=10^{11} \mathrm{~cm}^{-2}$ at the center of the QW at room temperature, we find, for our structure, a $\left\langle J_{z}\right\rangle$ comparable to the acoustic phonon contribution and, therefore, much less than the LO phonon contribution.

\section{APPENDIX E: REMARK ON THE ACTIVATION ENERGY OF THE THERMIONIC CURRENT}

We should note that there is an ambiguity regarding the activation energy in the scattering-assisted thermionic current. Formally speaking, since the electrons participating in the thermionic current are governed by the Boltzmann tail of their distribution function, imposing a lower bound to their possible in-plane wavevector immediately results in thermal activation of the thermionic current. In the static (charged impurities) or quasi-static (LA phonons) case, the activation energy is $V_{b}-\mu_{\mathrm{QW}}$ and a semilog plot of $J_{2}$ as a function of $1 / T$ would reveal the expected activation energy. In the case of the LO phonon-assisted thermionic current, the activation energy is reduced to $V_{b}-\mu_{\mathrm{QW}}-$ $\hbar \omega_{\text {LO }}$. Notably, however, plotting $J_{2}$ versus $1 / T$ would not necessarily reveal this reduced activation energy. This is because the number of scatterers, $n_{\mathrm{LO}}$, is exponentially dependent upon $T$ at low temperatures. Therefore, at low temperatures, the apparent activation energy is almost equal to $V_{b}-\mu_{\mathrm{QW}}$, while around room temperature and above the apparent activation energy gradually approaches $V_{b}-\mu_{\mathrm{QW}}-\hbar \omega_{\mathrm{LO}}$
[1] S. G. Kandlikar, Review and projections of integrated cooling systems for three-dimensional integrated circuits, J. Electron. Packag. 136, 024001 (2014).

[2] G. Pennelli, Review of nanostructured devices for thermoelectric applications, Beilstein J. Nanotechnol. 5, 1268 (2014).

[3] J. O. Sofo and G. D. Mahan, Optimum bandgap of a thermoelectric material, Phys. Rev. B 49, 4565 (1994).

[4] M. M. Leivo, J. P. Pekola, and D. V. Averin, Efficient peltier refrigeration by a pair of normal metal/insulator/superconductor junctions, Appl. Phys. Lett. 68, 1996 (1996).

[5] H. J. Goldsmid, Thermoelectric Refrigeration (Plenum, New York, 1964).

[6] G. Benenti, G. Casati, K. Saito, and R. S. Whitney, Fundamental aspects of steady-state conversion of heat to work at the nanoscale, Phys. Rep. 694, 1 (2017).

[7] A. Ziabari, M. Zebarjadi, D. Vashaee, and A. Shakouri, Nanoscale solid-state cooling: A review, Rep. Prog. Phys. 79, 095901 (2016).

[8] A. Shakouri, E. Y. Lee, D. L. Smith, V. Narayanamurti, and J. E. Bowers, Thermoelectric effects in submicron heterostructure barriers, Microscale Thermophys. Eng. 2, 37 (1998).

[9] R. Kim, C. Jeong, and M. S. Lundstrom, On momentum conservation and thermionic emission cooling, J. Appl. Phys. 107, 054502 (2010).

[10] G. D. Mahan, Thermionic refrigeration, J. Appl. Phys. 76, 4362 (1994).

[11] G. N. Hatsopoulos and J. Kave, Measured thermal efficiencies of a diode configuration of a thermo electron engine, J. Appl. Phys. 29, 1124 (1958).

[12] A. Shakouri and J. E. Bowers, Heterostructure integrated thermionic coolers, Appl. Phys. Lett. 71, 1234 (1997).

[13] T. Zeng and G. Chen, Energy conversion in heterostructures for thermoionic, Microscale Thermophys. Eng. 4, 39 (2000).

[14] G. D. Mahan, J. O. Sofo, and M. Bartkowiak, Multilayer thermionic refrigerator and generator, J. Appl. Phys. 83, 4683 (1998).

[15] G. D. Mahan and L. M. Woods, Multilayer Thermionic Refrigeration, Phys. Rev. Lett. 80, 4016 (1998).

[16] J. Zhang, N. Anderson, and K. M. Lau, Al/sub0.10/Ga/ sub 0.90/As-GaAs microcoolers, IEEE Electron Device Lett. 25, 345 (2004).

[17] D. Vashaee and A. Shakouri, Improved Thermoelectric Power Factor in Metal-Based Superlattices, Phys. Rev. Lett. 92, 106103 (2004).

[18] K. A. Chao, M. Larsson, and A. G. Mal'shukov, Roomtemperature semiconductor heterostructure refrigeration, Appl. Phys. Lett. 87, 022103 (2005).

[19] A. Yangui, M. Bescond, T. Yan, N. Nagai, and K. Hirakawa, Evaporative electron cooling in asymmetric double barrier semiconductor heterostructures, Nat. Commun. 10, 4504 (2019)

[20] M. Bescond, D. Logoteta, F. Michelini, N. Cavassilas, T. Yan, A. Yangui, M. Lannoo, and K. Hirakawa, Thermionic cooling devices based on resonant-tunneling AlGaAs/GaAs heterostructure, J. Phys.: Condens. Matter 30, 064005 (2018). 
[21] M. Bescond and K. Hirakawa, High-Performance Thermionic Cooling Devices Based on Tilted-Barrier, Phys. Rev. Appl. 14, 064022 (2020).

[22] S. Ray, P. Ruden, V. Sokolov, R. Kolbas, T. Boonstra, and J. Williams, Resonant tunneling transport at $300 \mathrm{~K}$ in GaAsAlGaAs quantum wells grown by metalorganic chemical vapor deposition, Appl. Phys. Lett. 48, 1666 (1986).

[23] T. J. Shewchuk, P. C. Chapin, and P. D. Coleman, Resonant tunneling oscillations in a GaAs- $\mathrm{Al}_{\mathrm{x}} \mathrm{Ga}_{1-\mathrm{x}} \mathrm{As}$ heterostructure at room temperature, Appl. Phys. Lett. 46, 508 (1985).

[24] S. M. Sze and K. K. Ng, Physics of Semiconductor Devices, 3rd ed. (Wiley, New York, 2007).
[25] S. Datta, Quantum Transport: Atom to Transistor (Cambridge University Press, New York, 2005).

[26] B. Jonsson and S. T. Eng, Solving the schrodinger equation in arbitrary quantum-well potential profiles using the transfer matrix method, IEEE J. Quantum Electron. 26, 2025 (1990).

[27] S. Datta, Electronic Transport in Mesoscopic Systems (Cambridge University Press, Cambridge, UK, 1995), pp. 257.

[28] C. R. Crowell, The Richardson constant for thermionic emission in Schottky barrier diodes, Solid-State Electron. 8, 395 (1965). 